Received: 19 April 2017

Accepted: 2 November 2017

Published online: 21 November 2017

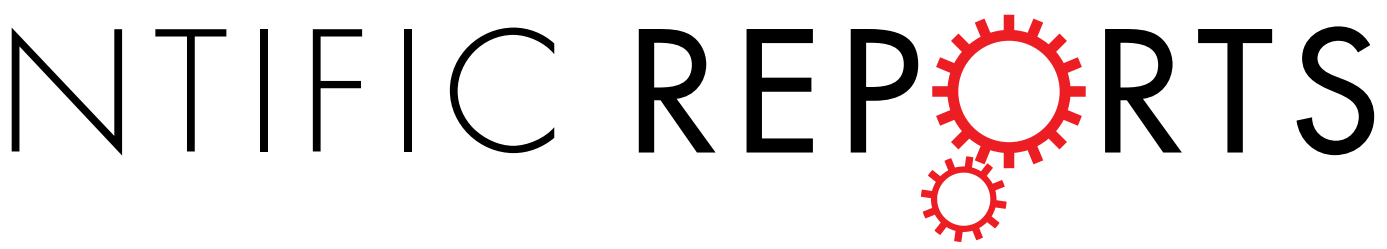

\title{
OPEN Association of serum sodium and risk of all-cause mortality in patients with chronic kidney disease: A meta-analysis and
} sysematic review

\author{
Liguang Sun ${ }^{1}$, Yue Hou ${ }^{2}$, Qingfei Xiao² \& Yujun Du ${ }^{2}$
}

Studies on the association of dysnatraemia with all-cause mortality risk in chronic kidney disease (CKD) patients have yielded inconsistent results. This meta-analysis aimed to evaluate the association of hyponatremia or hypernatremia with all-cause mortality risk in CKD patients. An electronic literature search was performed in Web of Science, Pubmed and Embase databases from inception to March 2017 for available observational studies evaluating the association of dysnatraemia with all-cause mortality risk in CKD patients. Pooled hazard risk (HR) with $95 \%$ confidence interval (Cl) was calculated for hyponatremia or hypernatremia vs. normonatremia. Seven studies that enrolled 742,979 CKD patients were identified. Baseline hyponatremia ( $\mathrm{HR} 1.34 ; 95 \% \mathrm{Cl}: 1.15-1.57)$, and not hypernatremia (HR 1.12; 95\%: $\mathrm{Cl} 0.93-1.34$ ), was independently associated with increased risk of all-cause mortality, when compared than the normonatremia category. In time-dependent analyses, both time-averaged hyponatremia (HR 1.65; 95\% Cl: 1.27-2.15) and hypernatremia (HR 1.41; 95\% Cl: $1.20-1.65$ ) had a higher independent risk of all-cause mortality. Furthermore, subgroup analyses by type of patients, study design, sample size and follow-up duration revealed similar results across most of these analyses. Baseline hyponatremia and time-dependent hyponatremia or hypernatremia were independently associated with increased all-cause mortality risk in CKD patients.

Chronic kidney disease (CKD) is an increasing global public health concern ${ }^{1}$. End-stage renal disease (ESRD) is the chronic and progressive decline in kidney function. More than two million people suffer from ESRD worldwide $^{2}$. Furthermore, the number of patients with ESRD receiving maintenance hemodialysis or peritoneal dialysis continues to increase worldwide ${ }^{3}$. Given that the mortality rate of CKD patients with or without dialysis is unacceptably high ${ }^{4,5}$, early risk stratification for mortality is crucial in these populations.

Dysnatremia is the most common electrolyte abnormality in clinical practice. Clinically, normal serum sodium level in humans ranges within $135-144 \mathrm{mmol} / \mathrm{L}^{6}$. Patients with CKD tend to develop dysnatremia mainly due to their diminished ability to maintain water homeostasis ${ }^{7}$. Hyponatremia and hypernatremia are relatively frequent electrolyte abnormalities in patients with advancing stages of $\mathrm{CKD}$, who are undergoing dialysis ${ }^{8}$. Several ${ }^{9-17}$ but not all ${ }^{18,19}$ epidemiologic studies reported that hyponatremia is associated with increased all-cause mortality in no-dialysis CKD and maintenance dialysis patients. Similarly, the association of hypernatremia with all-cause mortality risk has also yielded inconsistent results ${ }^{9,11-14,19}$.

To the best of our knowledge, no meta-analysis has addressed the association of baseline and time-dependent dysnatremia with subsequent all-cause mortality risk among CKD patients. Given the conflicting findings in these available studies, we conducted a meta-analysis to investigate whether dysnatremia (hyponatremia and hypernatremia) was an independent predictor of all-cause mortality in CKD patients with or without dialysis.

${ }^{1}$ Institute of Immunology, The First Hospital of Jilin University, 130021, Changchun, China. ${ }^{2}$ Department of Nephrology, The First Hospital of Jilin University, 130021, Changchun, China. Correspondence and requests for materials should be addressed toY.D. (email: duyujunjl@yeah.net) 


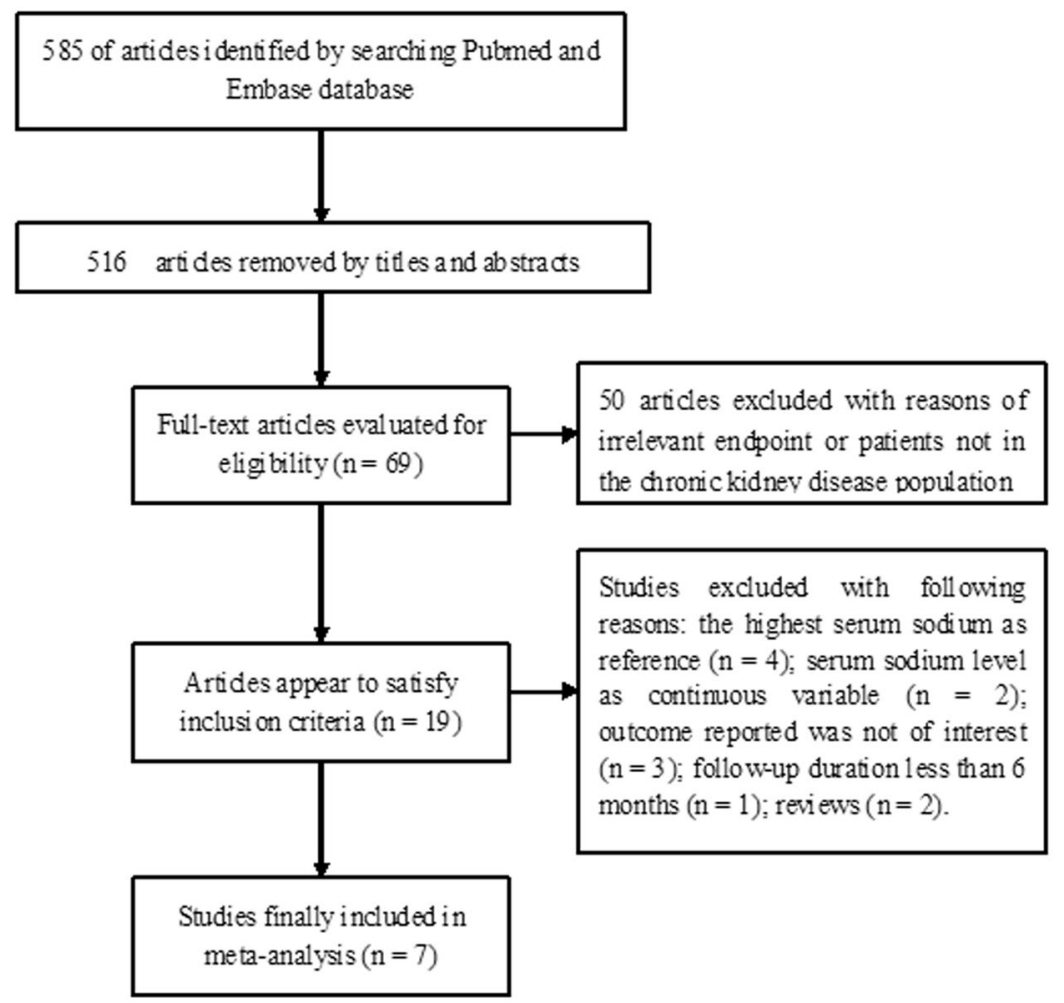

Figure 1. Flow chart of the study selection process.

\begin{abstract}
Results
Literature search and study characteristics. The initial literature search yielded 585 articles. Among these retrieved articles, 578 articles were excluded for different reasons (Fig. 1). Thus, seven studies ${ }^{9-14,19}$ that comprised of 742,979 CKD patients were included in the meta-analysis. Table 1 presents the baseline characteristics of the included studies. Among the seven articles, four studies ${ }^{9,11,14,19}$ were limited to non-dialysis CKD patients, two studies ${ }^{10,12}$ enrolled hemodialysis patients, and one study ${ }^{13}$ included peritoneal dialysis patients. Furthermore, five studies ${ }^{9,10,12-14}$ were conducted in the United States, Korea ${ }^{19}$ and the United Kingdom ${ }^{11}$, in which each country contributed one study. All seven studies followed an observational design, in which four studies were retrospective cohorts ${ }^{9,12-14}$ and three studies were prospective cohorts ${ }^{11,19}$. Furthermore, four studies ${ }^{9-11,14,19}$ defined hyponatremia as serum sodium $<136 \mathrm{mmol} / \mathrm{L}$ and three studies ${ }^{10,12,13}$ defined hyponatremia as serum sodium $<135 \mathrm{mmol} / \mathrm{L}$. The cut-off definition of hypernatremia was $\geq 144 \mathrm{mmol} / \mathrm{L}$ and $\geq 145 \mathrm{mmol} / \mathrm{L}$ in these included studies. All included studies were deemed as high quality, with a rank of 6-8 stars in the Newcastle-Ottawa Scale (NOS) (Table 2).
\end{abstract}

The association of hyponatremia with all-cause mortality. All the included studies ${ }^{9-14,19}$ evaluated the association of baseline hyponatremia and risk of all-cause mortality. In the time-dependent analyses of hyponatremia, six studies ${ }^{9,11-14,19}$ assessed this association. As shown in Fig. 2A, there was significant heterogeneity across these included studies $\left(I^{2}=92.9 \%, P<0.001\right)$. The meta-analysis revealed that patients with baseline hyponatremia were associated with a higher risk of all-cause mortality (hazard ratio [HR]: 1.34; $95 \%$ confidence interval [CI]: 1.15-1.57) compared with patients with normonatremia in a random effects model. Furthermore, there was no evidence of publication bias according to Begg's test $(P=0.368)$ and Egger's test $(P=0.151)$. The sensitivity analysis, in which an individual study was remove at a time, revealed that pooled HR varied within 1.321.40 and low $95 \%$ CI varied within 1.11-1.33. Similarly, patients with time-averaged hyponatremia was associated with higher risk of all-cause mortality (HR: 1.65; 95\% CI: 1.27-2.15; Fig. 2B) compared with patients with normonatremia in a random effects model, and there was significant heterogeneity across studies $\left(I^{2}=92.8 \% ; P<0.001\right)$. Begg's test $(P=1.000)$ and Egger's test $(P=0.117)$ did not reveal any evidence of publication bias. The sensitivity analysis indicated that pooled HR varied within $1.54-1.78$ and low $95 \%$ CI varied within $1.16-1.41$.

The association of hypernatremia and all-cause mortality. Six studies ${ }^{9,11-14,19}$ reported the association of baseline and time-averaged hypernatremia with all-cause mortality risk. The meta-analysis revealed that patients with baseline hypernatremia were not significantly associated with increased risk of all-cause mortality (HR: 1.12; 95\% CI: 0.93-1.34; Fig. 3A) compared with patients with normonatremia in a random effects model. Furthermore, there was significant heterogeneity across these included studies $\left(I^{2}=78.6 \% ; P<0.001\right)$. Moreover, Begg's test $(P=0.707)$ and Egger's test $(P=0.523)$ suggested no evidence of publication bias. The sensitivity analysis revealed that pooled HR varied within $1.06-1.17$ and low $95 \%$ CI varied within $0.88-0.99$. In 
Study

$\operatorname{HR}(95 \% \mathrm{Cl}) \quad$ Weight

A.Baseline hyponatremia

Kovesdy et al (2012)

Chiu et al (2012)

Nigwekar et al (2013)

Han et al (2015)

Rhee et al (2016)

Ravel et al (2016)

Huang et al (2016)

Subtotal (l-squared $=92.9 \%, p=0.000$ )

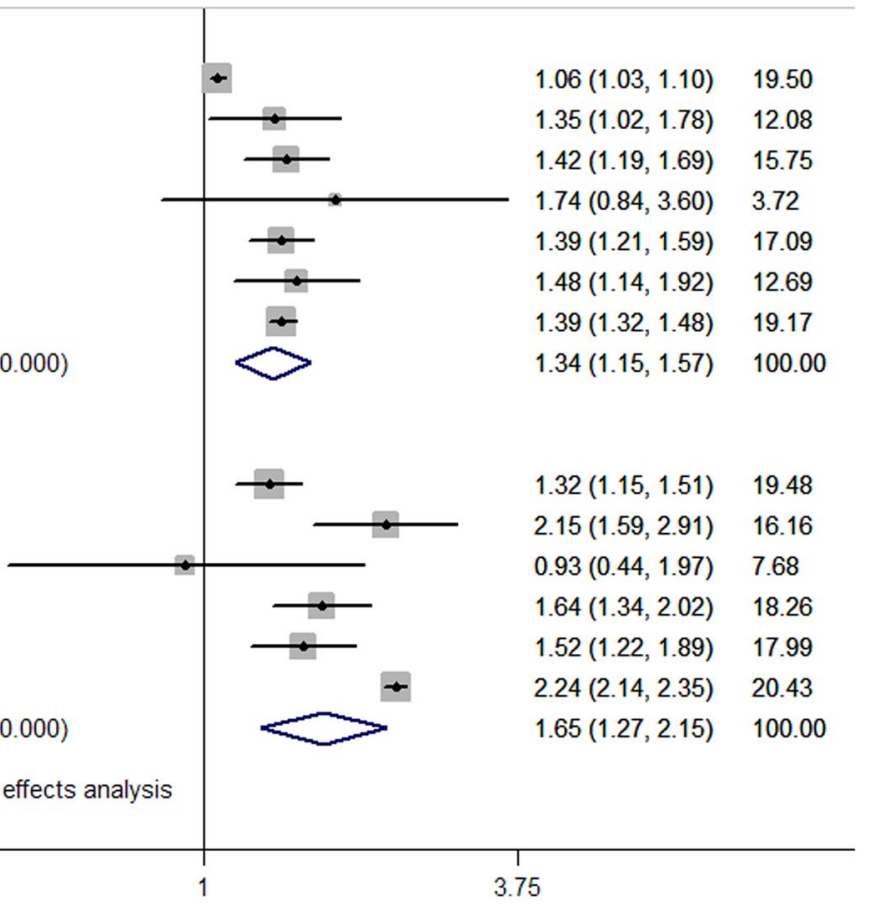

B.Time-averaged hyponatremia

Kovesdy et al (2012)

Chiu et al (2012)

Han et al (2015)

Rhee et al (2016)

Ravel et al (2016)

Huang et al (2016)

Subtotal (I-squared $=92.8 \%, p=0.000)$

3.75

NOTE: Weights are from random effects analysis

.28

Figure 2. Forest plots showing HR and 95\% CI of all-cause mortality comparing baseline hyponatraemia (A) or time-averaged hyponatraemia (B) to normonatremia in a random effect model.

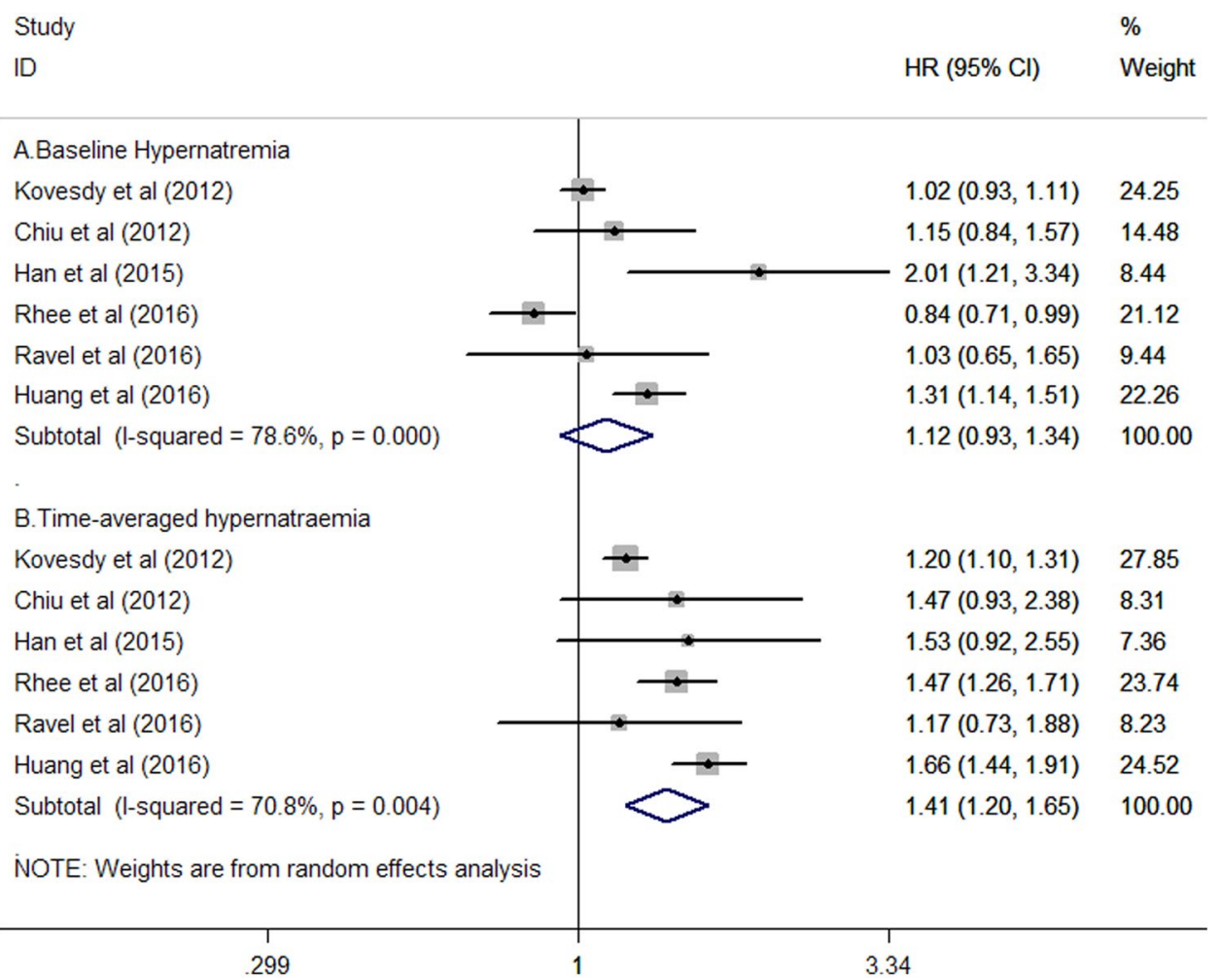

Figure 3. Forest plots showing HR and $95 \%$ CI of all-cause mortality comparing baseline hypernatraemia (A) or time-averaged hypernatraemia (B) to normonatremia in a random effect model. 


\begin{tabular}{|c|c|c|c|c|c|c|c|c|c|c|}
\hline Study/year & Country & Design & $\begin{array}{l}\text { Type of } \\
\text { patients }\end{array}$ & Baseline eGFR & $\begin{array}{l}\text { Sample size } \\
\text { (\% male) }\end{array}$ & $\begin{array}{l}\text { Age/range } \\
\text { Mean } \pm \text { SD }\end{array}$ & $\begin{array}{l}\text { Dysnatraemia } \\
\text { definition/number }\end{array}$ & $\begin{array}{l}\text { Number of death/HR } \\
(95 \% \mathrm{CI})\end{array}$ & $\begin{array}{l}\text { Follow-up } \\
\text { duration }\end{array}$ & Adjustment for covariates \\
\hline $\begin{array}{l}\text { Kovesdy et } \\
\text { al. } 2012^{9}\end{array}$ & USA & $\begin{array}{l}\text { Retrospective } \\
\text { cohort study }\end{array}$ & $\begin{array}{l}\text { Non-dialysis } \\
\text { CKD }\end{array}$ & $\begin{array}{l}55.2 \pm 19.3 \mathrm{ml} / \\
\mathrm{min} / 1.73 \mathrm{~m}^{2}\end{array}$ & $\begin{array}{l}655,493 \\
(97.2)\end{array}$ & $73.9 \pm 9.8$ & $\begin{array}{l}\mathrm{Na}<136 \mathrm{mmol} / \mathrm{L} \\
(85,855) ; \\
\mathrm{Na}>145 \mathrm{mmol} / \mathrm{L} \\
(350)\end{array}$ & \begin{tabular}{|l|} 
Total death: 193,956 \\
Baseline-Na 1.06 (1.03- \\
1.10); L 1.02(0.93-1.11); H \\
TA-Na 1.32(1.15-1.51); L \\
$1.20(1.10-1.31) ; \mathrm{H}$
\end{tabular} & 5.5 years & $\begin{array}{l}\text { Age, gender, race, geographic location, } \\
\text { DM, CVD, CHF, liver disease, } \\
\text { malignancy, depression, CCI, SBP, } \\
\text { eGFR, serum albumin, AKP, AST, } \\
\text { ALT, total bilirubin, hemoglobin, } \\
\text { glucose, and WBC }\end{array}$ \\
\hline $\begin{array}{l}\text { Nigwekar } \\
\text { et al. } 2013^{10}\end{array}$ & USA & $\begin{array}{l}\text { Prospective } \\
\text { cohort study }\end{array}$ & HD & $\begin{array}{l}10.5 \pm 6.2 \mathrm{ml} / \\
\mathrm{min} / 1.73 \mathrm{~m}^{2}\end{array}$ & $6,053(54.4)$ & $62.5 \pm 15.2$ & $\begin{array}{l}\mathrm{Na}<135 \mathrm{mmol} / \mathrm{L} \\
(775)\end{array}$ & $\begin{array}{l}\text { Total death: } 965 \text { Baseline- } \\
\text { Na 1.42(1.19-1.69) }\end{array}$ & 12 months & $\begin{array}{l}\text { Age, race, sex, DM, hypertension, } \\
\text { CAD, catheter access, facility } \\
\text { mortality statistic, BMI, serum } \\
\text { albumin, bicarbonate level. }\end{array}$ \\
\hline \multirow[t]{7}{*}{$\begin{array}{l}\text { Han et al. } \\
2015^{19}\end{array}$} & Korea & $\begin{array}{l}\text { Prospective } \\
\text { cohort study }\end{array}$ & $\begin{array}{l}\text { Non-dialysis } \\
\text { CKD }\end{array}$ & $\begin{array}{l}25.5 \pm 10.7 \mathrm{ml} / \\
\mathrm{min} / 1.73 \mathrm{~m}^{2}\end{array}$ & $2,141(55.2)$ & $63.5 \pm 14.9$ & $\begin{array}{l}\mathrm{Na} \leq 135 \mathrm{mmol} / \mathrm{L} \\
(135) ; \\
\mathrm{Na} \geq 144 \mathrm{mmol} / \mathrm{L} \\
(350)\end{array}$ & Total death: 182 & 1.8 years & $\begin{array}{l}\text { Age, gender, race, eGFR, SBP, } \\
\text { hemoglobin, CVD, serum albumin }\end{array}$ \\
\hline & & & & & & & & Baseline-Na & & \\
\hline & & & & & & & & $1.74(0.84-3.60) ; \mathrm{L}$ & & \\
\hline & & & & & & & & 2.01(1.21-3.34); H & & \\
\hline & & & & & & & & TA-Na & & \\
\hline & & & & & & & & 0.93 (0.44-1.97); L & & \\
\hline & & & & & & & & $1.53(0.92-2.55) ; \mathrm{H}$ & & \\
\hline \multirow[t]{7}{*}{$\begin{array}{l}\text { Chiuet al. } \\
2016^{11}\end{array}$} & UK & $\begin{array}{l}\text { Prospective } \\
\text { cohort study }\end{array}$ & $\begin{array}{l}\text { Non-dialysis } \\
\text { CKD }\end{array}$ & $\begin{array}{l}32.8 \pm 15.9 \mathrm{ml} / \\
\mathrm{min} / 1.73 \mathrm{~m}^{2}\end{array}$ & $2,093(62.6)$ & $56-75$ & $\begin{array}{l}\mathrm{Na} \leq 135 \mathrm{mmol} / \mathrm{L} \\
(142)\end{array}$ & Total death: 684 & 41 months & $\begin{array}{l}\text { Age, gender, smoking, DM, previous } \\
\text { MI, heart failure, SBP, eGFR, serum } \\
\text { albumin, use of renin-angiotensin } \\
\text { blocker or diuretics }\end{array}$ \\
\hline & & & & & & & $\begin{array}{l}\mathrm{Na} \geq 145 \mathrm{mmol} / \mathrm{L} \\
(134)\end{array}$ & Baseline-Na & & \\
\hline & & & & & & & & $1.35(1.02-1.78) ; \mathrm{L}$ & & \\
\hline & & & & & & & & $1.15(0.84-1.57) ; \mathrm{H}$ & & \\
\hline & & & & & & & & TA-Na & & \\
\hline & & & & & & & & $2.15(1.59-2.91) ; \mathrm{L}$ & & \\
\hline & & & & & & & & $1.47(0.93-2.38) ; \mathrm{H}$ & & \\
\hline \multirow[t]{7}{*}{$\begin{array}{l}\text { Rhee et al. } \\
2016^{12}\end{array}$} & USA & $\begin{array}{l}\text { Retrospective } \\
\text { study }\end{array}$ & HD & - & $27,180(57)$ & $63 \pm 15$ & $\begin{array}{l}\mathrm{Na}<136 \mathrm{mmol} / \mathrm{L} \\
(2,501)\end{array}$ & Total death: 7,562 & 1.4 years & $\begin{array}{l}\text { Age, sex, race/ethnicity, primary } \\
\text { insurance, vascular access, } \\
\text { comorbidities, IDWG, Kt/V, residual } \\
\text { urea clearance, BMI, serum albumin, } \\
\text { creatinine, total iron binding capacity, } \\
\text { ferritin, iron saturation, bicarbonate, } \\
\text { PTH, calcium, phosphorus, } \\
\text { hemoglobin, glucose, WBC, BUN, and } \\
\text { normalized protein catabolic rate. }\end{array}$ \\
\hline & & & & & & & $\begin{array}{l}\mathrm{Na} \geq 144 \mathrm{mmol} / \mathrm{L} \\
(549)\end{array}$ & Baseline-Na & & \\
\hline & & & & & & & & 1.39 (1.21-1.59); L & & \\
\hline & & & & & & & & $0.84(0.71-0.99) ; \mathrm{H}$ & & \\
\hline & & & & & & & & TA-Na & & \\
\hline & & & & & & & & 1.64(1.34-2.02); L & & \\
\hline & & & & & & & & $1.47(1.26-1.71) ; \mathrm{H}$ & & \\
\hline \multirow[t]{7}{*}{$\begin{array}{l}\text { Ravel et al. } \\
2016^{13}\end{array}$} & USA & $\begin{array}{l}\text { Retrospective } \\
\text { study }\end{array}$ & PD & - & $4,687(55)$ & $58 \pm 15$ & $\begin{array}{l}\mathrm{Na}<136 \mathrm{mmol} / \mathrm{L} \\
(399)\end{array}$ & Total death: 649 & 11.9 months & $\begin{array}{l}\text { Age, sex, race/ethnicity, primary } \\
\text { insurance, baseline comorbidities, } \\
\text { serum albumin, creatinine, total } \\
\text { iron binding capacity, calcium, } \\
\text { phosphorus, PTH, ferritin, iron } \\
\text { saturation, hemoglobin, WBC, } \\
\text { peritoneal Kt/v, renal Kt/V, use of } \\
\text { automated PD during the baseline } \\
\text { quarter or anytime }\end{array}$ \\
\hline & & & & & & & $\begin{array}{l}\mathrm{Na} \geq 144 \mathrm{mmol} / \mathrm{L} \\
(170)\end{array}$ & Baseline- $\mathrm{Na}$ & & \\
\hline & & & & & & & & $1.48(1.14-1.92) ; \mathrm{L}$ & & \\
\hline & & & & & & & & $1.03(0.65-1.65) ; \mathrm{H}$ & & \\
\hline & & & & & & & & TA-Na & & \\
\hline & & & & & & & & 1.52(1.22-1.89); L & & \\
\hline & & & & & & & & $1.17(0.73-1.88) ; \mathrm{H}$ & & \\
\hline \multirow[t]{7}{*}{$\begin{array}{l}\text { Huang et } \\
\text { al. } 2017^{14}\end{array}$} & USA & $\begin{array}{l}\text { Retrospective } \\
\text { study }\end{array}$ & $\begin{array}{l}\text { Non-dialysis } \\
\text { CKD }\end{array}$ & $\begin{array}{l}48.0 \pm 10.2 \mathrm{ml} / \\
\mathrm{min} / 1.73 \mathrm{~m}^{2}\end{array}$ & $\begin{array}{l}45,333 \\
(44.6)\end{array}$ & $71.9 \pm 11.9$ & $\begin{array}{l}\mathrm{Na}<136 \mathrm{mmol} / \mathrm{L} \\
(3,626)\end{array}$ & Total death: 11,715 & 3.6 years & $\begin{array}{l}\text { Age, gender, smoking, BMI } \\
\text { group, eGFR, DM, hypertension, } \\
\text { cerebrovascular disease, CAD, CHF, } \\
\text { hyperlipidemia, malignancy, ACEI/ } \\
\text { ARB, beta blocker, diuretics, albumin, } \\
\text { hemoglobin, serum bicarbonate and } \\
\text { liver disease }\end{array}$ \\
\hline & & & & & & & $\begin{array}{l}\mathrm{Na}>145 \mathrm{mmol} / \mathrm{L} \\
(532)\end{array}$ & Baseline-Na & & \\
\hline & & & & & & & & $1.39(1.32-1.48) ; \mathrm{L}$ & & \\
\hline & & & & & & & & $1.31(1.14-1.51) ; \mathrm{H}$ & & \\
\hline & & & & & & & & TA-Na & & \\
\hline & & & & & & & & 2.24 (2.14-2.35); L & & \\
\hline & & & & & & & & $1.66(1.44-1.91) ; \mathrm{H}$ & & \\
\hline
\end{tabular}


Table 1. Summary of clinical studies included in meta-analysis. Abbreviations: HD, hemodialysis; PD, peritoneal dialysis; L, low; $\mathrm{H}$, high; $\mathrm{HR}$, hazard ratio; $\mathrm{CI}$, confidence interval; CVD, cardiovascular disease; $\mathrm{CAD}$, coronary artery disease; $\mathrm{CHF}$, congestive heart failure; DM, diabetes mellitus; BMI, body mass index; $\mathrm{CKD}$, chronic kidney disease; MI, myocardial infarction; eGFR, estimated glomerular filtration rate; WBC, white blood cell count; CCI, Charlson Comorbidity Index; BUN, blood urea nitrogen; PTH, parathyroid hormone; TC, total cholesterol; TG, triglyceride; SBP, systolic blood pressure; AST, aspartate aminotransferase; ALT, alanine aminotransferase; AKP, alkaline phosphatase; TA-Na,time-averaged serum sodium; ACEI, angiotensin-converting enzyme inhibitor; $\mathrm{ARB}$, angiotensin receptor blocker.

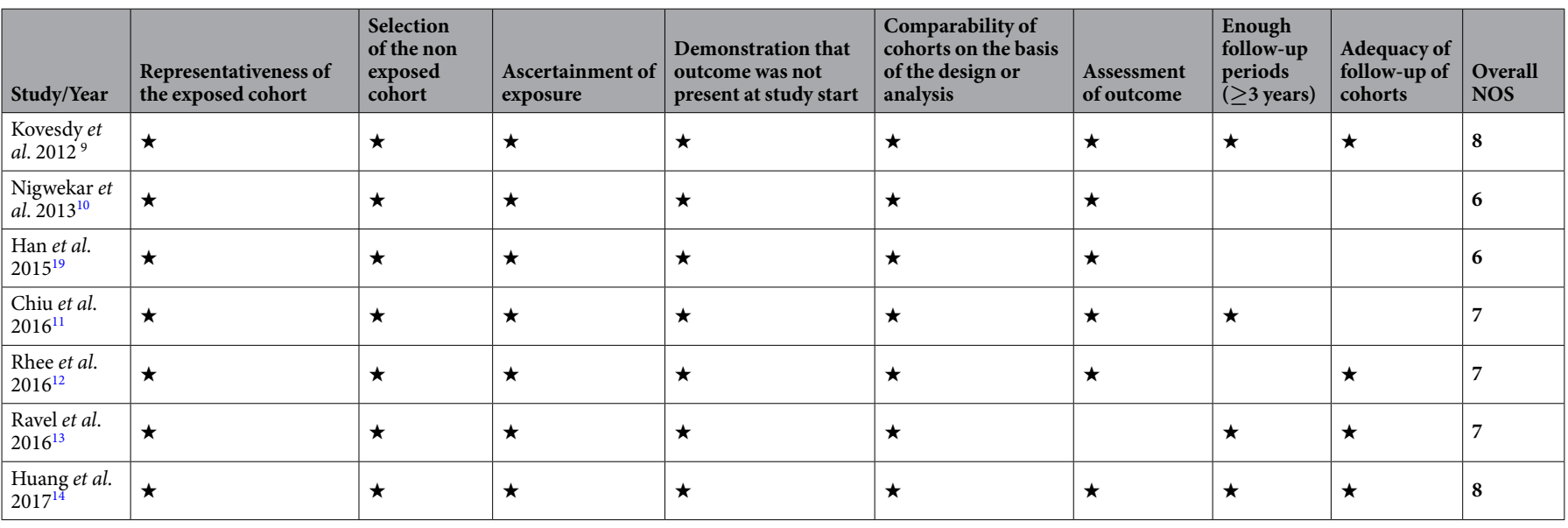

Table 2. quality assessment of studies included in meta-analysis

the time-dependent analysis of hypernatremia, patients with time-averaged hypernatremia was associated with higher risk of all-cause mortality (HR: 1.41 ; 95\% CI: 1.20-1.65; Fig. 3B) compared with patients with normonatremia in a random effects model, and there was substantial heterogeneity across these studies $\left(I^{2}=70.8 \%\right.$; $P=0.004)$. Furthermore, there was no evidence of publication bias based on the results of Begg's test $(P=1.000)$ and Egger's test $(P=0.522)$. The sensitivity analysis indicated that pooled HR varied within $1.32-1.54$ and low 95\% CI varied within 1.13-1.40.

Subgroup analyses. The stratified analysis revealed similar results across most of the subgroups, indicating a consistent association between dysnatremia and all-cause mortality (Tables 3 and 4 ).

\section{Discussion}

This is the first meta-analysis that investigated the association of dysnatremia with all-cause mortality risk. From the seven included observational studies, we found that baseline hyponatremia and time-dependent hyponatremia or hypernatremia were independently associated with increased risk of all-cause mortality in CKD patients. The risk of all-cause mortality for CKD patients who have time-dependent hyponatremia or hypernatremia increased by $41 \%$ and $65 \%$, respectively; while the risk of all-cause mortality for CKD patients who had hyponatremia at baseline increased by $34 \%$. However, the association of baseline hypernatremia with all-cause mortality was not statistically significant. Taken together, our results suggest that the association of serum sodium level with all-cause mortality appeared to exhibit a U-shaped trend in CKD patients. Therefore, dysnatremia may have potential in mortality risk stratification among these patients.

A previous meta-analysis revealed that moderate hyponatremia was associated with an increased 1.60 -fold risk of overall mortality ${ }^{20}$. However, this meta-analysis enrolled patients with myocardial infarction, heart failure, cirrhosis, pulmonary infection, or mixed diseases, and did not concentrate on CKD patients. Our meta-analysis was specially focused on CKD patients. We found that both hyponatremia and hypernatremia were strongly associated with higher risk of all-cause mortality when analyzed in a time-dependent manner. However, the combined risk estimate was not statistically significant when measured as a single baseline sodium level. Particularly, time-averaged hypernatremia was more strongly associated with all-cause mortality than single baseline hypernatremia. Water and sodium removal were almost exclusively determined by the dialysis procedure in ESRD patients. CKD patients undergoing dialysis are prone to develop dysnatremia. Therefore, the severity of the kidney disease may affect the mortality associated with dysnatraemia. In our subgroup analyses, patients undergoing dialysis exhibited a relatively higher all-cause mortality (HR: 1.27 vs. 1.41) associated with baseline hyponatremia, compared with patients with non-dialysis CKD. In contrast, the stage of CKD did not appear to affect the mortality associated with hypernatremia.

The prevalence of hyponatremia was significantly higher at all stages of CKD than the prevalence of hypernatremia ${ }^{6}$. A number of studies ${ }^{15-17,21}$ that examined the association of serum sodium with all-cause mortality risk have focused largely on hyponatremia. Irrespective of the severity of kidney disease, all studies found that hyponatremia was associated with increased risk of all-cause mortality. Each $4 \mathrm{mmol} / \mathrm{L}$ increase in baseline sodium level was associated 


\begin{tabular}{|c|c|c|c|c|}
\hline Subgroup & No. of studies & Pooled HR & 95\%CI & Heterogeneity between studies \\
\hline \multicolumn{5}{|c|}{ 1. Baseline hyponatraemia } \\
\hline \multicolumn{5}{|c|}{ Sample sizes } \\
\hline$\geq 10,000$ & 3 & 1.26 & $1.02-1.57$ & $\mathrm{p}<0.001 ; \mathrm{I}^{2}=97.3 \%$ \\
\hline$<10,000$ & 4 & 1.43 & $1.26-1.62$ & $\mathrm{p}=0.915 ; \mathrm{I}^{2}=0.0 \%$ \\
\hline \multicolumn{5}{|l|}{ Study design } \\
\hline Prospective & 3 & 1.41 & $1.22-1.63$ & $\mathrm{p}=0.810 ; \mathrm{I}^{2}=0.0 \%$ \\
\hline Retrospective & 4 & 1.3 & $1.07-1.58$ & $\mathrm{p}<0.001 ; \mathrm{I}^{2}=96.1 \%$ \\
\hline \multicolumn{5}{|l|}{ Follow-up duration } \\
\hline$\geq 2$ years & 3 & 1.24 & $0.99-1.56$ & $\mathrm{p}<0.001 ; \mathrm{I}^{2}=97.0 \%$ \\
\hline$<2$ years & 4 & 1.42 & $1.28-1.56$ & $\mathrm{p}=0.921 ; \mathrm{I}^{2}=0.0 \%$ \\
\hline \multicolumn{5}{|l|}{ Type of patients } \\
\hline Dialysis & 3 & 1.41 & $1.28-1.56$ & $\mathrm{p}=0.914 ; \mathrm{I}^{2}=0.0 \%$ \\
\hline Non-dialysis CKD & 4 & 1.27 & $1.02-1.59$ & $\mathrm{p}<0.001 ; \mathrm{I}^{2}=95.6 \%$ \\
\hline \multicolumn{5}{|c|}{ 2. Time-averaged hyponatraemia } \\
\hline \multicolumn{5}{|c|}{ Sample sizes } \\
\hline$\geq 10,000$ & 3 & 1.7 & $1.17-2.48$ & $\mathrm{p}<0.001 ; \mathrm{I}^{2}=96.5 \%$ \\
\hline$<10,000$ & 3 & 1.61 & $1.14-2.29$ & $\mathrm{p}=0.057 ; \mathrm{I}^{2}=65.1 \%$ \\
\hline \multicolumn{5}{|l|}{ Study design } \\
\hline Prospective & 2 & 1.52 & $0.68-3.42$ & $\mathrm{p}=0.042 ; \mathrm{I}^{2}=75.8 \%$ \\
\hline Retrospective & 4 & 1.66 & $1.21-2.26$ & $\mathrm{p}<0.001 ; \mathrm{I}^{2}=95.4 \%$ \\
\hline \multicolumn{5}{|l|}{ Follow-up duration } \\
\hline$\geq 2$ years & 3 & 1.84 & $1.24-2.73$ & $\mathrm{p}<0.001 ; \mathrm{I}^{2}=96.1 \%$ \\
\hline$<2$ years & 3 & 1.55 & $1.33-1.80$ & $\mathrm{p}=0.349 ; \mathrm{I}^{2}=5.0 \%$ \\
\hline \multicolumn{5}{|l|}{ Type of patients } \\
\hline Dialysis & 2 & 1.58 & $1.36-1.84$ & $\mathrm{p}=0.620 ; \mathrm{I}^{2}=0.0 \%$ \\
\hline Non-dialysis CKD & 4 & 1.68 & $1.16-2.44$ & $\mathrm{p}<0.001 ; \mathrm{I}^{2}=94.7 \%$ \\
\hline
\end{tabular}

Table 3. Subgroup analysis of hyponatraemia and all-cause mortality. Abbreviations: CKD, chronic kidney disease; HR, hazard ratio; CI, confidence interval; NOS, Newcastle-Ottawa Scale.

with $19-28 \%$ lower risk of all-cause mortality in maintenance hemodialysis patients ${ }^{22,23}$. As for the noted U-shape association of sodium level with mortality in CKD patients, elevated serum sodium levels also conferred to higher risk of all-cause mortality. The above mentioned studies selected the highest sodium level as the reference control. Thus, the association between hyponatremia and mortality in these studies might have been underestimated.

The exact mechanism of dysnatraemia in all-cause mortality risk in CKD patients remains unclear. Kidneys are responsible for maintaining water homeostasis, and CKD could magnify the effect of dysnatremias on their clinical consequences ${ }^{24,25}$. Both hyponatremia and hypernatremia can have direct adverse effects on the function of various organs, including the brain, heart, or musculoskeletal system; and subsequently, increase mortality.

For patients with renal disease, hyponatremia at baseline and time-dependent hyponatremia or hypernatremia had higher risk of mortality. Dysnatremia may be a potential target for correction for clinicians. However, whether correct dysnatraemia could improve outcomes should be evaluated in future studies.

Several limitations of this meta-analysis should be acknowledged. First, the number of included studies was relatively limited. Thus, the results of these subgroup analyses may not be robust. Second, there was statistical heterogeneity in the quantitative pooling outcome. Potential explanations for heterogeneity included the severity of the disease, follow-up duration and study design. Third, the measurement of serum sodium levels at a single time point could have resulted in the misclassification of dysnatremia due to the fluctuation of sodium values between dialysis sessions. However, time-averaged sodium analysis further confirmed the association of dysnatremia with all-cause mortality. A mean value of several monthly measurements of sodium may have provided more accurate results of dysnatremia. Fourth, the adjusted confounding factors were different across these included studies, and the lack of adjustment for these confounding factors may have slightly overestimated the risk estimate. Fifth, the lack of information on the dialysis regimen of these included studies was a potential limitation. Finally, this meta-analysis could not distinguish the effect of dysnatremia on peritoneal dialysis and hemodialysis.

In conclusion, baseline hyponatremia and time-dependent hyponatremia/hypernatremia are independently associated with increased all-cause mortality risk in CKD patients. This meta-analysis suggests the U-shaped association of dysnatremia with all-cause mortality risk in CKD patients. However, whether correcting dysnatremia can reduce mortality risk in CKD patients needs to be investigated in the randomized controlled trials.

\section{Methods}

Literature Search. The present meta-analysis was conducted in accordance with the checklists of the Metaanalysis Of Observational Studies in Epidemiology statement ${ }^{26}$. Two authors (LG Sun and Y Hou) independently searched the Web of Science, PubMed and Embase databases for available observational studies from inception 


\begin{tabular}{|c|c|c|c|c|}
\hline Subgroup & No. of studies & Pooled HR & 95\%CI & Heterogeneity between studies \\
\hline \multicolumn{5}{|c|}{ 1. Baseline hypernatraemia } \\
\hline \multicolumn{5}{|c|}{ Sample sizes } \\
\hline$\geq 10,000$ & 3 & 1.04 & $0.84-1.30$ & $\mathrm{p}<0.001 ; \mathrm{I}^{2}=88.1 \%$ \\
\hline$<10,000$ & 3 & 1.30 & $0.91-1.86$ & $\mathrm{p}=0.117 ; \mathrm{I}^{2}=53.5 \%$ \\
\hline \multicolumn{5}{|l|}{ Study design } \\
\hline Prospective & 2 & 1.46 & $0.85-2.52$ & $\mathrm{p}=0.066 ; \mathrm{I}^{2}=70.3 \%$ \\
\hline Retrospective & 4 & 1.04 & $0.86-1.86$ & $\mathrm{p}=0.001 ; \mathrm{I}^{2}=82.2 \%$ \\
\hline \multicolumn{5}{|l|}{ Follow-up duration } \\
\hline$\geq 2$ years & 3 & 1.15 & $0.95-1.39$ & $\mathrm{p}=0.012 ; \mathrm{I}^{2}=77.3 \%$ \\
\hline$<2$ years & 3 & 1.15 & $0.70-1.89$ & $\mathrm{p}=0.008 ; \mathrm{I}^{2}=80.9 \%$ \\
\hline \multicolumn{5}{|l|}{ Type of patients } \\
\hline Dialysis & 2 & 0.86 & $0.74-1.01$ & $\mathrm{p}=0.419 ; \mathrm{I}^{2}=0.0 \%$ \\
\hline Non-dialysis CKD & 4 & 1.23 & $0.99-1.52$ & $\mathrm{p}=0.003 ; \mathrm{I}^{2}=78.8 \%$ \\
\hline \multicolumn{5}{|c|}{ 2. Time-averaged hypernatraemia } \\
\hline \multicolumn{5}{|c|}{ Sample sizes } \\
\hline$\geq 10,000$ & 3 & 1.42 & $1.16-1.75$ & $\mathrm{p}<0.001 ; \mathrm{I}^{2}=87.8 \%$ \\
\hline$<10,000$ & 3 & 1.37 & $1.04-1.82$ & $\mathrm{p}=0.707 ; \mathrm{I}^{2}=0.0 \%$ \\
\hline \multicolumn{5}{|l|}{ Study design } \\
\hline Prospective & 2 & 1.5 & $1.06-2.12$ & $\mathrm{p}=0.910 ; \mathrm{I}^{2}=0.0 \%$ \\
\hline Retrospective & 4 & 1.39 & $1.15-1.68$ & $\mathrm{p}=0.001 ; \mathrm{I}^{2}=82.0 \%$ \\
\hline \multicolumn{5}{|l|}{ Follow-up duration } \\
\hline$\geq 2$ years & 3 & 1.42 & $1.08-1.85$ & $\mathrm{p}=0.001 ; \mathrm{I}^{2}=86.6 \%$ \\
\hline$<2$ years & 3 & 1.45 & $1.26-1.66$ & $\mathrm{p}=0.650 ; \mathrm{I}^{2}=0.0 \%$ \\
\hline \multicolumn{5}{|l|}{ Type of patients } \\
\hline Dialysis & 2 & 1.44 & $1.24-1.66$ & $\mathrm{p}=0.368 ; \mathrm{I}^{2}=0.0 \%$ \\
\hline Non-dialysis CKD & 4 & 1.43 & $1.13-1.81$ & $\mathrm{p}=0.002 ; \mathrm{I}^{2}=80.3 \%$ \\
\hline
\end{tabular}

Table 4. Subgroup analysis of hypernatraemia and all-cause mortality. Abbreviations: CKD, chronic kidney disease; HR, hazard ratio; CI, confidence interval; NOS, Newcastle-Ottawa Scale.

to March 2017. The following search terms were used: (chronic kidney disease OR end-stage renal disease OR hemodialysis OR peritoneal dialysis) AND (sodium OR hyponatraemia OR hypernatremia OR dysnatraemia) AND (mortality OR death). In addition, a manual search of the reference lists of all relevant articles was performed to identify any additional eligible publications.

Study Selection. Studies that satisfied the following criteria were eligible: (1) prospective or retrospective observational studies; (2) study populations that comprised of dialysis or non-dialysis CKD patients; (3) the exposure was baseline and time-averaged hyponatremia or hypernatremia; (4) the outcome measure was all-cause mortality; (5) the reported multiple adjusted HR and 95\% CI compared hyponatremia or hypernatremia with normonatremia. Exclusion criteria: (1) studies that used the highest serum sodium level as reference controls; (2) serum sodium levels were used as the continuous variable; (3) studies that had a follow-up duration of less than six months.

Data extraction and quality assessment. Two authors independently abstracted the following information into standardized forms: surname of the first author, publication year, country of origin, study design, sample size, percentage of male patients, age at enrollment, baseline estimated glomerular filtration rate (eGFR), hyponatremia or hypernatremia definition and number of patients, number of deaths, the most fully adjusted risk estimate, follow-up duration, and adjustment for potential cofounders. Any differences in opinion in the data extraction were resolved by discussion. In evaluating for methodological quality, the NOS was used for these cohort studies $^{27}$. The NOS was based on the following three aspects: selection, comparability and outcome. When using this scale, a total score of $\geq 7$ stars was deemed of high quality.

Data analysis. The pooled risk estimate was calculated through the category of hyponatremia or hypernatremia $v s$. normonatremia in these individual studies. Heterogeneity was explored using the Cochrane $Q$ test and $I^{2}$ statistic. Statistical heterogeneity was set at an $I^{2}$ statistic of $\geq 50 \%$ and/or $Q$ test of $P<0.10$. In the presence of statistical heterogeneity, studies were pooled using a random effect model. Otherwise, a fixed-effect model was used. Subgroup analyses were performed according to the type of patients (dialysis $v s$. non-dialysis CKD), study design (prospective $v s$. retrospective), sample size $(\geq 10,000 v s .<10,000)$ and follow-up duration $(\geq 2$ years $v s$. $<2$ years). A sensitivity analysis was conducted to investigate the impact of these individual studies on the overall results by removing one study at each turn. Potential publication bias was explored using Begg's test and Egger's test. All statistical analyses were performed using STATA software 12.0 (Stata Corp, College Station, Texas). 


\section{References}

1. Hill, N. R. et al. Global Prevalence of Chronic Kidney Disease - A Systematic Review and Meta-Analysis. PLoS One 11, e0158765 (2016).

2. Robinson, B. M. et al. Factors affecting outcomes in patients reaching end-stage kidney disease worldwide: differences in access to renal replacement therapy, modality use, and haemodialysis practices. Lancet 388, 294-306 (2016).

3. Thomas, B. et al. Maintenance Dialysis throughout the World in Years 1990 and 2010. J Am Soc Nephrol 26, 2621-33 (2015).

4. Cheung, A. K. et al. Atherosclerotic cardiovascular disease risks in chronic hemodialysis patients. Kidney Int 58, 353-62 (2000).

5. Herzog, C. A. et al. Cardiovascular disease in chronic kidney disease. A clinical update from Kidney Disease: Improving Global Outcomes (KDIGO). Kidney Int 80, 572-86 (2011).

6. Kovesdy, C. P. Significance of hypo- and hypernatremia in chronic kidney disease. Nephrol Dial Transplant 27, 891-8 (2012).

7. Combs, S. \& Berl, T. Dysnatremias in patients with kidney disease. Am J Kidney Dis 63, 294-303 (2014).

8. Zhang, R., Wang, S., Zhang, M. \& Cui, L. Hyponatremia in patients with chronic kidney disease. Hemodial Int 21, 3-10 (2017).

9. Kovesdy, C. P. et al. Hyponatremia, hypernatremia, and mortality in patients with chronic kidney disease with and without congestive heart failure. Circulation 125, 677-84 (2012).

10. Nigwekar, S. U., Wenger, J., Thadhani, R. \& Bhan, I. Hyponatremia, mineral metabolism, and mortality in incident maintenance hemodialysis patients: a cohort study. Am J Kidney Dis 62, 755-62 (2013).

11. Chiu, D. Y., Kalra, P. A., Sinha, S. \& Green, D. Association of serum sodium levels with all-cause and cardiovascular mortality in chronic kidney disease: Results from a prospective observational study. Nephrology (Carlton) 21, 476-82 (2016).

12. Rhee, C. M. et al. Pre-dialysis serum sodium and mortality in a national incident hemodialysis cohort. Nephrol Dial Transplant 31, 992-1001 (2016).

13. Ravel, V. A. et al. Serum sodium and mortality in a national peritoneal dialysis cohort. Nephrol Dial Transplant (2016).

14. Huang, H. et al. Associations of dysnatremias with mortality in chronic kidney disease. Nephrol Dial Transplant 32, 1204-10 (2017).

15. Hecking, M. et al. Predialysis serum sodium level, dialysate sodium, and mortality in maintenance hemodialysis patients: the Dialysis Outcomes and Practice Patterns Study (DOPPS). Am J Kidney Dis 59, 238-48 (2012).

16. Perez-Garcia, R. et al. Hyponatraemia, mortality and haemodialysis: An unexplained association. Nefrologia 36, 42-50 (2016).

17. Sahin, O. Z. et al. The impact of low serum sodium level on mortality depends on glycemic control. Eur J Clin Invest 42, 534-40 (2012).

18. Chen, K. H., Chen, C. Y., Lee, C. C., Weng, C. M. \& Hung, C. C. Baseline hyponatremia does not predict two-year mortality in patients with chronic peritoneal dialysis. Ren Fail 36, 1371-5 (2014).

19. Han, S. W. et al. Serum sodium levels and patient outcomes in an ambulatory clinic-based chronic kidney disease cohort. Am J Nephrol 41, 200-9 (2015).

20. Corona, G. et al. Moderate hyponatremia is associated with increased risk of mortality: evidence from a meta-analysis. PLoS One $\mathbf{8}$, e80451 (2013).

21. Chang, T. I. et al. Hyponatremia as a predictor of mortality in peritoneal dialysis patients. PLoS One 9, e111373 (2014).

22. Waikar, S. S., Curhan, G. C. \& Brunelli, S. M. Mortality associated with low serum sodium concentration in maintenance hemodialysis. Am J Med 124, 77-84 (2011).

23. Mc Causland, F. R., Brunelli, S. M. \& Waikar, S. S. Dialysate sodium, serum sodium and mortality in maintenance hemodialysis. Nephrol Dial Transplant 27, 1613-8 (2012).

24. Mitch, W. E. \& Wilcox, C. S. Disorders of body fluids, sodium and potassium in chronic renal failure. Am J Med 72, 536-50 (1982).

25. Hoorn, E. J. \& Zietse, R. Hyponatremia and mortality: moving beyond associations. Am J Kidney Dis 62, 139-49 (2013).

26. Liberati, A. et al. The PRISMA statement for reporting systematic reviews and meta-analyses of studies that evaluate health care interventions: explanation and elaboration. J Clin Epidemiol 62, e1-34 (2009).

27. Wells, G. et al. The Newcastle-Ottawa Scale (NOS) for assessing the quality if nonrandomized studies in meta-analyses. http://www. ohri.ca/programs/clinical_epidemiology/oxford.asp (assessed August 20, 2016).

\section{Author Contributions}

L.G. Sun and Y. Hou made the literature research, extracted data, and conducted the statistical analysis. Q.F. Xiao drafted the manuscript and evaluated the study quality. Y.J. Du designed the study, interpreted the results, and approved the final version of this manuscript.

\section{Additional Information}

Competing Interests: The authors declare that they have no competing interests.

Publisher's note: Springer Nature remains neutral with regard to jurisdictional claims in published maps and institutional affiliations.

(c) (i) Open Access This article is licensed under a Creative Commons Attribution 4.0 International cc. License, which permits use, sharing, adaptation, distribution and reproduction in any medium or format, as long as you give appropriate credit to the original author(s) and the source, provide a link to the Creative Commons license, and indicate if changes were made. The images or other third party material in this article are included in the article's Creative Commons license, unless indicated otherwise in a credit line to the material. If material is not included in the article's Creative Commons license and your intended use is not permitted by statutory regulation or exceeds the permitted use, you will need to obtain permission directly from the copyright holder. To view a copy of this license, visit http://creativecommons.org/licenses/by/4.0/.

(C) The Author(s) 2017 